vol. $20-n^{\circ} 1 \mid 2004$

Numéro ouvert

\title{
Le traitement de l'islam au Canada. Tendances actuelles
}

Denise Helly

\section{(2) OpenEdition \\ Journals}

Édition électronique

URL : https://journals.openedition.org/remi/274

DOI : 10.4000/remi.274

ISSN : $1777-5418$

Éditeur

Université de Poitiers

Édition imprimée

Date de publication : 1 mai 2004

Pagination : 47-73

ISBN : 2-911627-36-9

ISSN : 0765-0752

Référence électronique

Denise Helly, «Le traitement de l'islam au Canada. Tendances actuelles », Revue européenne des migrations internationales [En ligne], vol. 20 - $\mathrm{n}^{\circ} 1$ | 2004, mis en ligne le 24 septembre 2008, consulté le 14 avril 2022. URL : http://journals.openedition.org/remi/274 ; DOI : https://doi.org/10.4000/remi.274

Ce document a été généré automatiquement le 14 avril 2022

(C) Université de Poitiers 


\title{
Le traitement de l'islam au Canada. Tendances actuelles
}

\author{
Denise Helly
}

1 Au Canada, la Loi sur le multiculturalisme (1988) reconnaît la diversité des Canadiens en termes de race, d'origine nationale ou ethnique et de religion et ce trait est une caractéristique fondamentale de la société et de l'État canadiens. Aussi la discrimination envers les personnes de confession musulmane est-elle un sujet d'intérêt depuis les attentats aux États-Unis le 11 septembre 2001, un intérêt d'autant plus grand que la visibilité de l'islam est un phénomène récent au Canada医; elle date des années 1990. Cette nouvelle visibilité tient à des polémiques autour du port du foulard islamique dans des écoles québécoises en 1994 et à la publication de données selon lesquelles la religion en croissance la plus rapide depuis 1991 est l'islam. En 2001, la population musulmane comprenait 579㡠000 personnes contre 253医000 en 1991 et la majorité vivait dans la région torontoise. Là, les personnes d'origine pakistanaise composaient le groupe le plus important alors que la région montréalaise constituait un second centre de concentration. Au total, 120医000 personnes se déclarant de confession musulmane et majoritairement d'origine arabe résidaient dans la métropole ${ }^{1}$.

2 Dans ce contexte, le propos de cet article est triple㹂: décrire les formes et l'étendue de la discrimination subie par les musulmans au Canada, notamment depuis le 11 septembre 2001, en repérer les fondements et, éventuellement, en expliciter la spécificité. Dans ce dessein, il faut préciser les définitions de la discrimination, dont celles qui sont données par la législation canadienne, car elles délimitent le débat sur la discrimination et les recours des individus contre celle-ci.

\section{Les définitions de la discrimination et le droit à l'égalité}

3 La Charte canadienne des droits et libertés incluse dans la Constitution de 1982 et de similaires chartes provinciales ${ }^{2}$ protègent les libertés fondamentales (conscience, 
religion, opinion, expression, réunion pacifique, association, défense) et les droits fondamentaux (vie et sécurité, sauvegarde de la dignité, de l'honneur et de la réputation, respect de sa vie privée, jouissance paisible de biens, non harcèlement, présomption d'innocence). Elles interdisent la discrimination fondée sur la race, la couleur, l'origine ethnique ou nationale, la religion, l'âge, le sexe, l'orientation sexuelle, un handicap mental ou physique, que ce soit en matière de libertés et droits fondamentaux comme de droits politiques, judiciaires, économiques et sociaux.

4 Le droit à l'égalité, protégé par ces documents est quadruple䜿: égalité devant la loi, égalité lors de l'application de la loi, égalité de protection par la loi et égal bénéfice de la loi. La notion d'égal bénéfice de la loi est importante, car elle vise à contrer une conception formelle de l'égalité. En effet, il est un principe juridique canadien[⿷匚⿱⿰㇒一母: : un traitement identique de tous par la loi peut provoquer une inégalité et le respect d'une véritable égalité exige souvent que des distinctions soient faites (Crépeau, 1996).

5 Dans un jugement charnière en 1989 (Andrews c. Law Society of British Columbia [1989] R.C.S. 143), la Cour suprême du Canada définit la discrimination comme « une distinction, intentionnelle ou non, mais fondée sur des motifs relatifs à des caractéristiques personnelles d'un individu ou d'un groupe d'individus, qui a pour effet d'imposer à cet individu ou à ce groupe des fardeaux, des obligations ou des désavantages non imposés à d'autres ou d'empêcher ou de restreindre l'accès aux

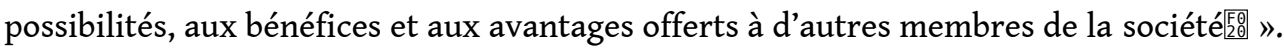
Selon cette définition, la discrimination est un déni d'égalité subi par un individu ou un groupe en raison d'un critère illicite de distinction. Elle peut être directe ou indirecte.

6 On parle de discrimination directe lorsqu'un ou plusieurs des critères illicites selon la loi sont explicitement invoqués pour dénier un droit ou une liberté. On parle de discrimination indirecte lorsqu'une mesure produit un effet inégalitaire pour un groupe de personnes identifiables selon un critère illicite (phénotype, origine culturelle, âge, genre, religion, handicap), sans que l'auteur de la mesure ait explicitement visé cet effet (Bosset, 1989, 1994医; Ledoyen, 1992). L'exemple souvent cité est l'exigence d'un poids ou d'une taille pour l'obtention d'un poste de policier ou de pompier, laquelle de fait exclut les membres de certaines minorités immigrées. La Cour suprême donna une valeur juridique à la discrimination indirecte, la jugeant en 1985 une « discrimination par effet préjudiciable ${ }_{20}^{[\mathrm{F}}$ » (Commission ontarienne des droits de la personne c. Simpson Sears Ltd [1985] 2 R.C.S. 536).

7 On parle encore de discrimination systémique quand des inégalités entre des groupes de personnes ne sont pas imputables à un facteur repérable mais relèvent d'un ensemble de facteurs, présents ou passés. Telle est la sous-représentation dans certaines occupations de personnes issues des minorités immigrées par rapport aux groupes dits majoritaires, les Canadiens d'ascendance britannique ou française. Aussi, la sous-représentation des membres des minorités racialisées, dites «minorités

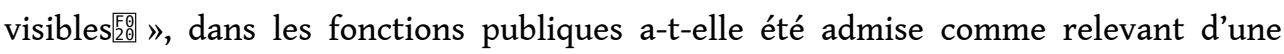
discrimination systématique passée et présente, car elle ne relève pas de caractéristiques admises comme sources de différenciation économique (niveau de scolarité, expérience de travail, durée de séjour pour les immigrés, connaissance des langues officielles). Des législations visent à la réduire, dont au niveau fédéral la Loi sur l'équité en emploi (1986), et au Québec les Programmes d'accès à l'égalité en emploi (1985) et la Loi 143 sur l'accès à l'égalité en emploi dans des organismes publics (2000). 
On distingue encore la discrimination selon sa source. On parle de discrimination institutionnalisée lorsque des lois et mesures publiques excluent intentionnellement des personnes de la jouissance d'un droit que les autres se voient reconnaître. Ce déni de droits ou libertés a, par exemple, existé de 1908 aux années 1960 quand des quotas très faibles étaient appliqués aux ressortissants de pays du Moyen-Orient ou en 1885 quand les immigrés chinois se virent imposer une taxe d'entrée au Canada. Enfin, on parle de discrimination voilée (Kunz, Milan et Schetagne, 2001), coutumière (Ledoyen, 1992) ou volontariste (Mc Andrew et Potvin, 1996) pour désigner des attitudes ou pratiques privées qui, à partir d'un critère illicite selon la loi, portent à exclure des personnes de sphères de la vie sociale quotidienne. Ces pratiques présentent la caractéristique d'être difficile à prouver, peu documentées et non chiffrées, et aussi de rarement donner lieu à des plaintes. Mais leurs effets sont observables, comme la faible présence de membres de certains groupes ethnoculturels dans des zones d'habitat, dans des associations et clubs et dans les réseaux sociaux d'autres groupes (collègues, voisins, amis, intermariages).

Dans le cas des personnes de confession musulmane, ces formes de discrimination peuvent être attestées dans des domaines et difficiles à confirmer dans d'autres.

\section{La discrimination directe ou le déni de libertés et droits fondamentaux}

\section{Par des personnes privées}

\section{Crimes haineux}

Des gestes hostiles à l'égard d'une personne ou d'un groupe en raison d'un attribut les définissant personnellement, soit des insultes publiques, une incitation publique à la haine, une attaque physique ou une attaque de biens, sont des atteintes aux droits à la dignité, la sécurité, l'intégrité et la jouissance paisible de biens et sont dénommés « crimes haineux娣 ». Depuis 1971, ils constituent au Canada des crimes ${ }^{3}$.

11 Cette forme de discrimination est peu documentée avant la guerre du Golfe (1991) quand les manifestations d'islamophobie commencent à être notées par des organisations islamiques et de protection des droits. Depuis septembre 2001, elles sont plus systématiquement enregistrées et attestent d'une multitude de crimes haineux dont les conséquences ont été parfois sérieuses selon les organisations communautaires. Peur et abattement ont suscité nombre d'appels d'urgence par des personnes anxieuses de savoir comment assurer leur sécurité personnelle. S'est manifestée notamment la crainte de montrer des signes perçus comme liés à la confession musulmane (vêtement, barbe, couvre-chef), de fréquenter les lieux de culte et les écoles musulmans, de s'absenter du travail lors de fêtes religieuses, d'être marginalisé socialement ou d'être soumis à une surveillance de la police fédérale ou du Service canadien du renseignement de sécurité.

Pour l'ensemble du Canada, le Congrès islamique canadien (CIC) signala 1 600医\% d'augmentation de crimes haineux à l'égard de personnes ou lieux musulmans de septembre 2001 à septembre 2002 (Media Communiqué, 10 mars 2003). Il avait reçu 11 plaintes relatives à de tels crimes l'année précédant les attentats contre 173 l'année les ayant suivis. Le rapport du Federal Bureau of Investigation (FBI) pour l'année 2001 fait 


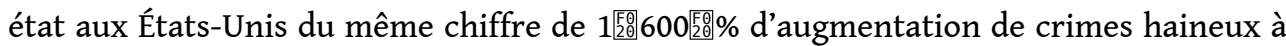

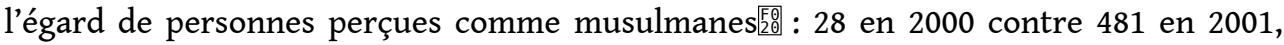
dont 3 meurtres et 35 incendies criminels (Abdelkarim, 2003[铝: 51).

Quant au Toronto Police Service Hate Crime Unit (2001澄 : 11 et 22), il nota une hausse

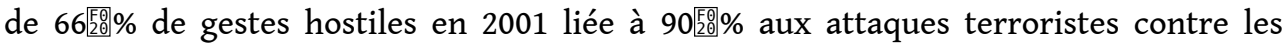
États-Unis, soit 121 des 338 crimes haineux commis durant l'année, majoritairement entre septembre et octobre 2001. De ces 121 actes, 16 furent des attaques physiques contre des personnes ou des biens, 39 des cas de troubles dits mineurs, 48 des menaces, et 57 visaient directement des musulmans ou l'islam ${ }^{4}$ (ibid.: 13). Comparativement, en 2001 on avait compté 58 crimes haineux envers des personnes d'origine juive, 53 envers des personnes de phénotype noir et 24 envers des personnes homosexuelles (ibid.医: 8 ) et, en 2000, un seul crime haineux envers une personne identifiée comme musulmane. Les services de police de trois autres villes canadiennes firent également état d'une recrudescence de crimes haineux de septembre à décembre 2001, tous reliés aux attentats aux États-Unis医 : 40 à Montréal, 24 à Calgary et 44 à Ottawa (Hussain, 2002區: 23). Dans ces trois villes, les cas avaient été rares avant septembre 2001. Enfin, entre le 11 septembre et le 15 novembre 2001, la section canadienne du Council on American Islamic Relations (CAIR) fut avisée de 110 incidents, dont 10 menaces de mort, 10 messages haineux, 33 agressions verbales et 13 voies de fait (Nimer, 2002).

Cependant, les statistiques en matière de harcèlement verbal, d'attaque physique et de menace de mort demeurent imprécises pour plusieurs raisons. Ces gestes ne donnent que rarement lieu à des plaintes des victimes et leurs témoins ne les rapportent pas. Ce dernier fait est connu. Selon la plus récente enquête en la matière, réalisée en 2002 en France, $48{ }_{[2]}^{\mathbb{E}} \%$ des Français interrogés se dirent prêts à signaler un comportement raciste à la police (Zappi, 2003). Lors de groupes de discussion organisés par le Conseil canadien des femmes musulmanes (Hussain, 2002医: 23) et regroupant 181 femmes, les participantes relatèrent 40 cas de crimes haineux (appels téléphoniques menaçants ou dégradants, insultes sur la rue, vandalismes, voies de fait), dont 2 seulement rapportés à la police. À Montréal, entre les 11 et 20 septembre 2001, le Service de police enregistra une douzaine de plaintes pour harcèlement verbal. Pourtant, entre septembre 2001 et septembre 2002, 83 crimes haineux furent dénombrés (Taillefer, 2002) et, selon les témoignages rapportés aux $\mathrm{ONG}$, les insultes fondées sur leurs origines à l'égard de Musulmans ou d'immigrés du Moyen-Orient augmentèrent dans la rue, les transports publics ou les milieux de travail. Il en fut de même dans tous les pays. Aux États-Unis, alors que le FBI faisait état de 481 crimes haineux en 2001, 1 1 Council on American Islamic Relations (CAIR) de septembre 2001 à février 2002 (Abdelkarim, 2003).

15 Un fait renforce la tendance à ne pas porter plainte aux autorités dans le cas de la population de confession musulmane canadienne. Sa structuration communautaire est à peine amorcée en dépit d'une multitude d'associations religieuses et séculières de vocations variées et les deux organisations pancanadiennes suivant le dossier des atteintes aux droits et libertés des personnes de confession musulmane, CAIR-CAN et le Congrès islamique canadien, disposent de peu de soutien et de moyens.

Enfin, autre facteur de l'imprécision des statistiques㷡: des services de police canadiens n'enregistrent pas les crimes haineux (Halifax) ou n'enregistrent pas l'origine ethnoculturelle des victimes (Windsor) ou leur religion (Hamilton, Calgary, Waterloo, Edmonton). De plus, les catégories utilisées pour identifier les victimes ou classer les 
crimes haineux varient. Le Toronto Police Service Hate Crime Unit, qui existe depuis 1993, utilise concurremment les catégories de Muslim, Pakistani, Middle Easterner, Somali, Arab and East Indian䃈; aussi est-il difficile de savoir si la religion musulmane, l'origine nationale ou culturelle ou le phénotype sont les raisons de crimes haineux. La même unité ne traite pas des insultes criées dans la rue comme des crimes haineux alors que les organisations musulmanes et B'nai Brith (The Gazette, 2003b) le font.

Quant aux attaques de lieux de culte musulmans, inexistantes auparavant, elles furent nombreuses au lendemain des attentats. CAIR-CAN (2002) en cite $12 \mathrm{du} 11$ septembre au 15 novembre 2001 pour l'ensemble du Canada (Hussain, 2002塸: 14) et, selon les témoignages de 181 femmes de confession musulmane résidant dans diverses régions canadiennes, au moins une attaque eut lieu contre un lieu de culte dans chaque ville canadienne entre septembre 2001 et juin 2002 (Hussain, 2002 㡠 : 15) 㡠; 16 étaient des attentats à la bombe. Une présence policière n'avait été assurée que durant quelques jours ou semaines après les attentats, devant les écoles musulmanes et les lieux de culte musulmans les plus importants du pays lors des prières du vendredi.

18 Au Canada comme dans les pays de l'Europe occidentale (Diène, 2003医: $:$ ), les crimes haineux diminuèrent en 2002 et après. Le Toronto Police Service Hate Crime Unit (2002医: 10 et 13) en recensa 10 en 2002 contre des «musulmans盛 》, auxquels doivent

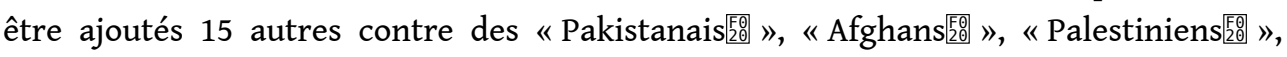

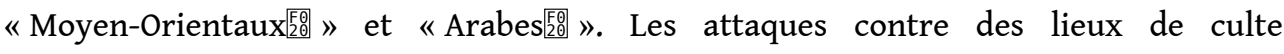
diminuèrent aussi, mais, récemment, en avril 2003, des graffitis haineux «Mort aux

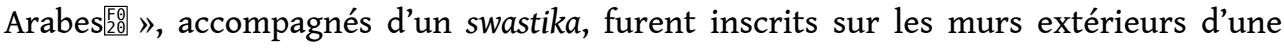
école (Écoles musulmanes de Montréal).

19 Cette baisse tiendrait selon des intervenants canadiens en milieu immigré à l'expérience et à l'organisation des autorités municipales, scolaires et policières en matière de gestion de conflits interethniques et au réseau de relations existant entre ces autorités, les organismes publics et les ONG communautaires intervenant en milieu ethnique. Dans les principales villes canadiennes, les autorités policières disposent d'unités de gestion de crise comprenant des représentants ethniques et ce lien fut mis à profit lors d'incidents violents. Par exemple, en septembre 2001 à Ottawa, lors du rouage de coups d'un adolescent de confession musulmane par des jeunes gens d'origine européenne, les corps municipaux et les ONG condamnèrent l'attaque et rallièrent l'opinion publique, enrayant, fut-il dit, toute reproduction de pareil geste. À l'opposé, à Hamilton (Ontario), lors de l'incendie total d'un temple sikh, confondu avec une mosquée, la situation s'envenima faute de contacts entre le corps de police et les communautés ethniques.

20 On observe qu'au Canada les crimes haineux ont surtout pris la forme d'insultes, de menaces et d'attaques de lieux de culte alors que les agressions physiques furent peu fréquentes. Deux agressions ont été violentes, l'une contre une famille d'immigrés pakistanais battue dans un parc de Montréal au printemps 2002 et l'autre contre l'adolescent sévèrement blessé à Ottawa en septembre 2001. Dans les deux cas, des jeunes d'origine européenne furent impliqués.

21 Selon le rapport spécial des Nations Unies (Diène, 2003覧: 2, 4), l'hostilité à l'égard des personnes arabes et en général de confession musulmane prit des formes différentes selon le pays: particulièrement contre des femmes portant le hidjab; multiplication de conflits sur

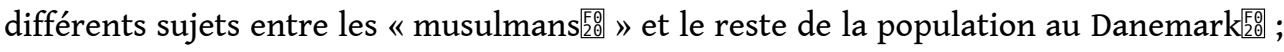


attaques de lieux de culte aux Pays-Bas (90 du 11 septembre au 2 octobre 2001 selon l'Association of Anti-discrimination Centers)疑; attaques à la bombe de mosquées en Australie et saccage d'une église libanaise maronite惯; multiplication d'actes de malveillance en France (169 déclarés en 2002 dont un tiers dans le Nord et en Île-deFrance (Zappi, 2003) 医; et nombreux cas de harcèlement verbal et d'attaques de personnes aux États-Unis.

\section{Discriminations sur le marché du travail}

Le seul diagnostic sur le racisme au Canada a été réalisé pour la province du Québec en 1996 (Mc Andrew et Potvin). Les auteurs conclurent que la discrimination était présente dans des milieux professionnels (médecine, soins infirmiers, droit, enseignement pré-universitaire, génie civil, travaux publics) et prenait la forme de stratégies d'évitement, de suivi incomplet de dossiers de candidature et de mise à distance des activités sociales courantes et des réseaux hors du milieu de travail (ibid. [.0日 : 55). La discrimination se manifestait de manière plus subtile pour les travailleurs non qualifiés des secteurs de services peu productifs, du commerce de détail et de gros, du textile et du vêtement. Recrutement préférentiel de membres de certains groupes culturels par des employeurs, difficultés de syndicalisation, disparités de salaire entre groupes culturels, exigence exagérée d'expérience de travail canadienne, déqualification très fréquente et tensions interethniques entretenues par les employeurs étaient des faits attestés, particulièrement dans le cas de nouveaux arrivants, des femmes et des jeunes des minorités dites visibles (ibid.员: 53-57). Mais, rédigé entre 1994 et 1996, ce diagnostic s'appuie sur des données des années 1980 et il distingue les groupes haïtien, jamaïcain, latino-américain et d'Asie de l'Est mais non ceux issus de pays musulmans, encore peu nombreux. Cette lacune existe pareillement

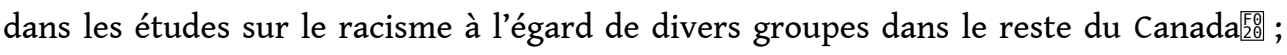
elles ne traitent pas des musulmans.

Deux enquêtes à l'échelle du Québec montrent que les mêmes obstacles et désavantages demeurent au début des années 2000 alors que le nombre de personnes de confession musulmane sur le marché du travail a significativement augmenté. Selon une enquête réalisée auprès de formateurs en employabilité d'ONG québécois, trois catégories de personnes ont des difficultés particulières à trouver un emploi䜿: «les personnes noires, d'origine arabe et “visiblement" musulmane㡠》 (Tadlaoui, 2002區: 20). La discrimination est directe et prend la forme de refus de prise en considération de curriculum vitae de personnes d'origine arabe ou de confession musulmane sous prétexte qu'elles ne participent pas à la vie de l'entreprise et que leurs habitudes sont trop éloignées de celles dites québécoises. La seconde enquête réalisée par la Ligue des droits et libertés du Québec (Lubuto Mutoo, 2001) avant septembre 2001 montre la force de ces préjugés䜿: des questionnaires sur la discrimination en emploi des «minorités visibles監 》 furent envoyés à 197 employeurs dans la ville de Québec, 19 seulement répondirent ${ }^{5}$ dont un tiers (35医\%) dit refuser d'employer un "Arabe臨》 ou un Maghrébin. Par ailleurs, des ONG québécoises œuvrant à l'insertion des minorités ethniques sur le marché du travail provincial ont reçu des appels d'employeurs leur demandant de ne pas référer « d'Arabes覧 » et, significativement, le Directeur de l'état civil du Québec mentionna une augmentation "phénoménale $F_{20}^{\text {F } ~ " ~ d e ~ " d e m a n d e s ~ d e ~}$

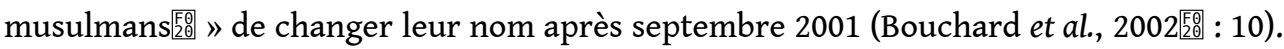
De plus, des postulants à un emploi ont été écartés à cause de leur accent en français, 


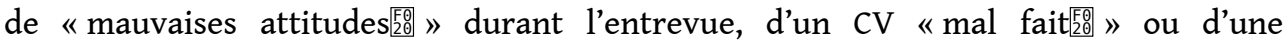
perception négative de leur groupe culturel ou racial par la clientèle dans le cas de services commerciaux. Ces formes de discrimination directe sont attestées par les témoignages d'émigrés des pays musulmans dans d'autres villes québécoises (Bel Hassen, 2002医: 12) et canadiennes (Ville d'Ottawa, 2002).

Une autre raison de la difficulté d'accès à un emploi des membres des «minorités visibles監 » est plus difficile à cerner mais réelle. Il s'agit de la non-appartenancee aux réseaux d'emploi vu qu'environ $80\left[\left[_{0} \%\right.\right.$ des emplois du secteur privé ne sont pas affichés pour le grand public. La pratique des employeurs qui consiste à recruter au sein de réseaux qu'ils connaissent est dénommée clonage (Luboto Mutoo, 2001)哰; elle tient souvent à un souci d'économie de temps et d'argent qu'accentuent l'ethnocentrisme, le racisme ou la xénophobie. Quant à la discrimination en milieu de travail, elle se manifeste sous la forme de menaces et de réflexions désobligeantes à l'égard de signes rapportés à la religion musulmane (foulard, vêtement, barbe), de congédiement pour avoir exprimé une opinion politique, le plus souvent sur une question relative au Moyen-Orient ou d'un refus d'ôter un signe vestimentaire comme le foulard (Lubuto Mutoo, 2001).

Les formes de la discrimination à l'emploi et dans le milieu de travail demeurent très peu documentées au Canada et elles donnent rarement lieu à de plaintes. De plus, les raisons invoquées pour un refus d'embauche ou un congédiement de membres de groupes culturels minoritaires ou racialisés s'enveloppent souvent d'arguments prétextes selon l'expression du Bureau International du Travail (1968) et les réelles raisons sont souvent difficiles à reconstituer.

Une autre forme d'atteinte à un droit économique a été constatée depuis septembre 2001. Des résidents portant le prénom de Osama virent leur compte en banque gelé de manière injustifiée ou des collègues tenter de les faire débaucher. Un documentaire en production retrace ces expériences. Intitulé «Being Osama區 », il est financé en partie par un organisme public québécois (SODEC) et sera diffusé par les principales chaînes de télévision canadiennes ( $\mathrm{CBC}, \mathrm{CTV}$ et Global) et par Al Jazeera (Montgomery, 2003).

\section{Discrimination en matière d'éducation}

Des incidents autour de la pratique musulmane dans le champ scolaire se sont produits uniquement au Québec et avant septembre 2001. Ils illustrèrent la présence d'une hostilité à l'égard de la culture musulmane de la part de parents d'élèves, d'enseignants ou de directeurs d'établissement. En 1988, un comité de parents d'une école montréalaise (Henri Beaulieu) refusa un cours d'arabe langue seconde, alors qu'existait un programme du ministère de l'Éducation du Québec à cet effet. La direction de l'école créa le cours mais les arguments des opposants révélèrent l'importance d'un stéréotype arabe qui commençait à devenir un stéréotype musulman : "L'enseignement de l'arabe

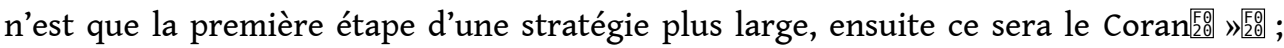
«Les garçons sont déjà assez machos comme ça, que va-t-on enseigner dans ce

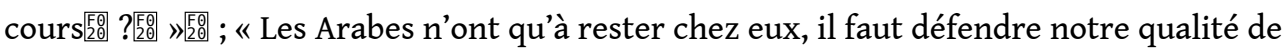

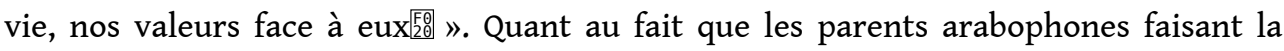
demande fussent surtout des Chrétiens et des mères scolarisées, il ne fut pas reconnu par les parents francophones ou présenté comme une stratégie afin de cacher des intentions réelles (« Ils mettent de l'avant les chrétiens et ces femmes-là, mais il ne faut 
pas s'y tromper 1991 quand, dans une autre école montréalaise, des parents de confession musulmane demandèrent un enseignement de la morale musulmane comme le permettent la législation provinciale et la Charte québécoise des droits de la personne. Aucune solution ne fut apportée vu l'opposition des parents d'autres confessions (Proulx, 1994). Enfin, en 1994-1995, douze élèves portant le foulard firent les manchettes des journaux. Après six mois de controverses, la Commission des droits de la personne du Québec émit un avis en 1995區: pareil usage devait être permis sous peine de porter atteinte aux droits des jeunes filles. Des mouvements nationalistes (Helle-Mai Lenk, 2000), féministes et laïque (Ciceri, 1999) protestèrent sans effet et la dispute publique cessa.

Contrairement à ce qui était attendu du milieu scolaire montréalais, les incidents racistes (crimes haineux, déni de droits) entre élèves ou entre élèves et personnel scolaire furent rares en septembre 2001 et après, et rapidement contrôlés. Dès la semaine des attentats et les semaines suivantes, des contacts furent établis par les corps de police avec des représentants d'organisations issues de la population

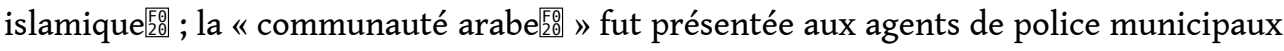
(patrouilleurs), aux intervenants socio-communautaires dans les écoles et aux directeurs d'école, des équipes de gestion de stress post-traumatique établies dans les écoles montréalaises et des locaux ouverts dans ces écoles et certaines universités pour les élèves et professeurs impliqués dans des incidents (Duchesne, 2001).

\section{La discrimination par des organismes gouvernementaux et leurs agents}

\section{Ciblage ethnique et atteintes aux libertés}

Une loi antiterrorisme (C-36) a été adoptée le 7 décembre $2001^{6}$ par le Parlement canadien. Elle impliqua la modification de vingt-deux lois canadiennes existantes (Jézéquel, 2002) dont le code criminel, la protection des renseignements personnels, l'accès à l'information et la loi de la preuve dont la Couronne n'est plus contrainte de transmettre tous les éléments. Elle crée de nouvelles infractions criminelles 医: facilitation [sic] de/et incitation à des actes terroristes ${ }^{7}$, affiliation à des organisations soupçonnées de contribuer à de pareils actes, transaction avec une entité terroriste entraînant la saisie de propriétés et de biens soupçonnés de servir à des activités terroristes ${ }^{8}$, annulation du statut d'organisation charitable à tout groupe finançant ou soupçonné de financer des activités terroristes. En matière de protection des libertés, cette loi accorde plus de pouvoirs à la police qui acquiert le droit d'opérer des perquisitions secrètes, d'étendre la période des écoutes électroniques, auparavant de six mois, d'écouter les communications d'une personne avec l'étranger sur simple décision du ministre de la Défense et sans contrôle judiciaire et, enfin, de créer un fichier des voyages aériens de tout Canadien, conservé pendant six ans. La police a aussi désormais les droits de détention préventive de 72 heures sans motif, d'enquête sans mandat et d'interrogatoire obligatoire devant un juge sous peine d'un an de prison. De ces mesures, deux sont révisables après cinq ans, celles qui portent sur la preuve et la détention préventive. Les autres sont permanentes. Cette loi demeure très critiquée par le Barreau canadien et les organisations de défense des droits.

Elle menace, en effet, les libertés individuelles de tout Canadien par ses procédures portant atteinte aux droits d'un accusé au silence et à la connaissance de l'accusation 
portée contre lui et elle vise directement les personnes de confession musulmane. Elle a deux conséquences pour celles-ci醩: leur ciblage par la police, notamment aux frontières (Hurst, 2002医; Makin, 2003), la tentative de collecte d'informations par le Service canadien du renseignement de sécurité (SCRS) et la police fédérale (GRC) auprès de personnes impliquées dans la vie de la communauté musulmane ou au statut d'immigration précaire (étudiants étrangers, demandeurs d'asile). Ces actions créent ou renforcent la suspicion dans l'opinion publique d'une présence significative de militants islamistes violents au sein de la population musulmane canadienne.

\section{La discrimination indirecte}

\section{La discrimination systémique}

31 La discrimination systémique des groupes ethnoculturels minoritaires s'alimente à diverses pratiques ${ }_{20.0}^{\left[F_{0}\right.}$ : refus d'embauche et de promotion, exigence de qualifications supérieures, rémunération plus faible. Elle s'accentue depuis 20 ans au Canada en dépit de multiples programmes du Programme du multiculturalisme pour la contrer. Selon les données de Statistique Canada, en 2000, les hommes immigrés obtenaient un salaire de 63,1 cents versus 1 医 $\$$ par les natifs de même niveau scolaire ${ }^{9}$ alors que ce rapport était de 71,6 cents en 1980 pour les hommes arrivés cette année-là. De plus, les hommes immigrés depuis dix ans recevaient en moyenne 79,8 cents comparativement à 1 凨\$ par

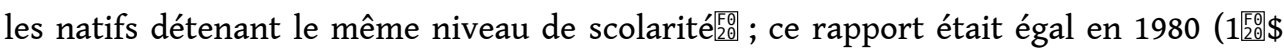
pour tous). Enfin, jusqu'au début des années 1980, les immigrés rattrapaient en dix ans le handicap de ne pas détenir d'expérience de travail canadienne, un argument utilisé depuis des lustres pour les sous-payer. Dans le cas des femmes, le rapport était et demeure plus défavorable. La situation des immigrés arrivés depuis les années 1980, dont une fraction importante est musulmane, apparait donc défavorable.

Une étude (Pendakur, 2000) montre aussi que les personnes d'origine non européenne subissaient entre 1971 et 1991 un désavantage certain sur le marché de l'emploi canadien, leur revenu était inférieur de 8 医\% comparativement à celui des personnes d'origine européenne, à âges et niveaux de scolarité égaux. Par ailleurs, le pourcentage de membres des «minorités visibles憕 》 qui possèdent un diplôme post-secondaire étant plus élevé que le pourcentage des autres Canadiens, on s'attendrait à ce que ce fait se reflète dans la répartition des occupations, mais il n'en est rien. Seuls les secteurs des affaires et des travaux publics montrent des taux d'emploi similaires entre groupes racialisés et européens (Kunz, Milan et Schetagne, 2001). Les secteurs de l'informatique et des technologies de pointe constituent les seules réelles mosaïques culturelles en ce qui concerne la composition de leur personnel. Enfin, au Québec, les

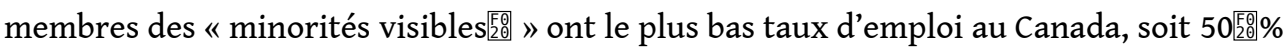
versus 70䂑\% (Kunz, Milan et Schetagne, 2001).

Toutefois, ces données ne permettent pas de savoir si et comment les personnes de confession musulmane subissent ces désavantages faute d'analyses statistiques ${ }^{10}$ prenant en compte la religion. On ne peut pas plus parler de discrimination systémique dans l'accès aux emplois des fonctions publiques car on ne dispose pas plus de statistiques tenant compte de la religion dans ce secteur. Il est cependant attesté que tous les groupes d'immigration récente se retrouvent sous représentés dans ce secteur. $\mathrm{Au}$ Québec, 2,1医\% des postes de la fonction publique sont actuellement occupés par des 
immigrés et leurs descendants et, selon le recensement de 1996, 5,9[医\% des postes de la fonction publique fédérale l'étaient par des membres des «minorités visibles 医》", soit très en deçà de leur poids dans la population canadienne (19\%) (Groupe de travail, 2000). Le même constat d'ignorance est à faire dans un domaine où la discrimination systémique peut être active, le marché du logement.

\section{Les négociations culturelles et l'obligation juridique d'accommodement raisonnable} peuvent concerner les pratiques diététiques de malades hospitalisés, de personnes incarcérées ou d'enfants placés en famille d'accueil, l'ouverture de salles de prières sur les lieux de travail et dans les écoles, les modes de punition des enfants qui mettent en jeu les lois de protection de la jeunesse et l'autorité parentale, le retrait des filles de l'école avant l'âge légal pour cause de mariage, ce qui contrevient à l'égalité des droits des femmes, le port d'un costume différent pour les filles lors des cours d'éducation physique ou encore le mode d'inhumation. Au Canada les demandes d'accommodement culturel ou religieux sont difficiles à ignorer parce que les droits des minorités religieuses sont fortement protégés par les Chartes des droits canadienne et provinciales. Toutefois, le groupe musulman apparaît plus ciblé quant à la fréquence et à l'ampleur des conflits autour d'ajustements culturels. Par exemple, les prescriptions religieuses des Témoins de Jéhovah sont plus contestables au plan de la réussite des élèves et de leur intégration psychosociale et contraires à la fonction éducative critique de l'école, mais leurs demandes d'accommodement n'atteignent jamais le degré de visibilité donnée à celles du groupe musulman (Mc Andrew, 2002㜯: 138).

d'accommodement raisonnable en cas de discrimination indirecte. La cause opposait une employée adventiste du Septième jour demandant de conserver son emploi à temps plein et le respect du repos du sabbat, et son employeur, Simpsons Sears Ldt., qui refusait sa demande. La Cour suprême estima qu'un accommodement devait réduire la discrimination subie par l'employée en raison de sa confession et précisa que la solution à trouver devait être raisonnable, c'est-à-dire qu'aucune contrainte excessive ne devait être imposée à l'employeur, soit un coût financier exagéré, des inconvénients importants, la réduction de normes de sécurité ou une atteinte aux droits d'autres employés et aux conventions collectives. Dans ce cas, la Cour estima que l'horaire de travail pouvait être aménagé. L'esprit de ce jugement s'applique à d'autres aspects du travail ainsi qu'à d'autres domaines, tel l'offre de services et de biens privés ou publics.

La notion d'accommodement raisonnable s'impose au Canada en vertu du texte législatif qui invoque la promotion d'une société pluraliste équitable (Loi sur le multiculturalisme canadien, Ottawa, 1988) et de l'article 27 de la Charte canadienne qui stipule que son interprétation doit tenir compte du patrimoine multiculturel canadien. À la différence des autres pays occidentaux, cette notion constitue une obligation

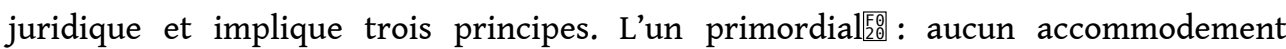
culturel ne peut porter atteinte aux droits et libertés d'une personne et aux lois fondamentales. Le respect de différences culturelles ne peut jamais contrevenir à la logique fondatrice du système juridique et politique canadien, le respect des droits individuels et la non-ingérence de l'État dans la sphère religieuse. D'ailleurs aucun 
droit culturel collectif qui permettrait de créer des enclaves fermées, tel le droit à des tribunaux séparés, n'est accordé aux minorités ethnoculturelles. Le respect des lois fondamentales donne lieu à d'autres pratiques dans des pays occidentaux. Depuis septembre 2002, en Flandres (non en région wallonne), au nom de l'équité des religions reconnues par la constitution belge, les écoliers de confession musulmane, juive ou chrétienne orthodoxe ont congé les jours de grande fête de leur culte (soit deux jours supplémentaires pour les Musulmans, dix-sept pour les Israélites, trois pour les Chrétiens orthodoxes).

L'autre principe veut que les solutions trouvées doivent maintenir un équilibre entre les droits des plaignants et que ceux-ci contribuent à les mettre à jour et à les appliquer. L'esprit de tout accommodement raisonnable est d'éviter la multiplication de litiges juridiques et de viser la réduction d'inégalités culturelles par la négociation.

De nombreux accommodements raisonnables ont été adoptés durant les années 1980 et 1990, notamment en faveur de minorités religieuses car, comme l'écrivit la Cour suprême dans le jugement de 1985医: «Un inconvénient minime est le prix à payer pour la liberté de religion dans une société multiculturelle国 》. Voici des exemples d'accommodement à Montréal, région de concentration des immigrés et de leurs

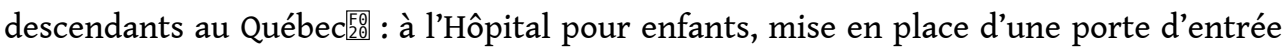
actionnée manuellement pour permettre aux Juifs hassidiques de visiter les malades le jour du sabbat医; ;ans des écoles publiques, création d'une journée pédagogique mobile pour que les enfants de religions orthodoxe, copte et catholique puissent célébrer le jour de Pâques à leur date respective ${ }_{20}^{\mathbb{E}}$; évitement d'interruption des services publics dans le quartier chinois durant le Nouvel An chinois (Conseil des relations interculturelles et de l'immigration, 1993)膯; droit, obtenu à la suite d'une plainte, pour un groupe hassidim de placer des fils d'erouv dans un quartier de la ville (Cour supérieure du Québec, juin 2001) ${ }^{11}$. Toutefois, le port du kirpan à l'école publique a suscité une âpre polémique en 2002 [F⿱ ${ }_{0}$; ce signe, a-t-il été statué, peut être porté si enveloppé d'un tissu le rendant inoffensif mais le jugement est actuellement devant la Cour d'appel du Québec.

Dans le cas de la population musulmane, avant les années 1980, une négociation a été menée à bien entre la Ville de Montréal et deux organisations (mosquée al Islam, sunnite医; Fondation internationale Az'zahra, chiite) pour l'ouverture d'un carré musulman dans deux cimetières et le droit de célébrer et d'enregistrer les mariages a été accordé à la mosquée al Islam. Puis, durant les années 1990 d'autres accommodements furent adoptés: de prière pour les employés de religion minoritaire et, devant le refus des fidèles musulmans de partager ce lieu avec des fidèles de religions non monothéistes (hindouisme, bouddhisme), ouverture d'un local spécial à leur intention䂑; dans les écoles publiques, droit pour les jeunes filles musulmanes de porter un costume plus pudique lors des cours d'éducation physique ${ }_{20}^{\text {F }}$; dans d'autres écoles allocation d'une période de deux heures pour permettre aux élèves musulmans de faire la prière $d u$ vendredi源; dans certaines unités du Canadien National, une grande entreprise canadienne, aménagement de l'horaire de travail pour créer trois périodes libres par jour pour les prières des employés musulmans: ${ }_{20}$; dans les piscines municipales, plage horaire de trois heures réservée aux jeunes musulmans et partagée entre garçons et filles[20 ; lieux de prières pour les étudiants musulmans dans deux universités. Des demandes d'accommodement n'eurent par contre aucune suite, comme l'ouverture de 
carrés musulmans dans les cimetières d'autres villes que Montréal, le refus d'un employé de la Sûreté du Québec de traduire l'enregistrement d'une conversation recueillie par écoute parce que sa religion lui interdisait toute délation ou encore le refus d'un employé du Service de police de Montréal d'imposer des amendes de stationnement pour motif de conscience ( ne pas faire de répression㵧 »). Et la polémique autour du port du foulard resurgit périodiquement, comme dernièrement à la rentrée scolaire de 2003.

Néanmoins, la situation depuis septembre 2001 ne se particularise pas par un nombre plus élevé de demandes, de refus ou d'acceptations d'accommodements en faveur de personnes musulmanes mais par quelques refus hostiles, appuyés d'arguments politiques comme au Québec l'invocation du principe de la "laïcité:[0] $~^{12}$ (demande d'une salle de prières par les étudiants musulmans de l'École de technologie supérieure, Montréal, 2003) et, surtout, par leur mention par la presse, un fait nouveau au Canada. La présence de l'islam est devenue un trait admis de la société canadienne.

\section{La discrimination coutumière}

41 La discrimination dite coutumière, voilée ou volontariste demeure difficile à prouver faute d'atteintes attestées à des libertés ou droits fondamentaux, mais des sondages d'opinion sur les attitudes à l'égard des musulmans la montrent à l'œuvre.

\section{Les attitudes}

Deux enquêtes passées montrent que ces personnes étaient victimes d'une perception défavorable. Selon la première, pancanadienne (Angus Reid, 1991), la presque totalité des répondants dirent se sentir plus à l'aise avec des natifs qu'avec des immigrés et encore moins à l'aise avec les membres des groupes, indien, pakistanais, sikh, noir antillais, arabe et musulman. Selon la seconde, québécoise (Joly et Dorval, 1993), les groupes ethniques les plus dévalorisés étaient les groupes pakistanais et jamaïcain en raison de leur anglophonie mais peut-être aussi, dans le premier cas, de la religion. Et, selon d'autres enquêtes annuelles pancanadiennes, les personnes rattachées à la religion musulmane étaient toujours celles avec lesquelles des Canadiens «ne se sentent pas à l'aise医 》.

Par ailleurs, des conflits ont montré l'existence d'un climat d'intolérance religieuse plus fort au Québec. Lors du conflit en 1994-1995 sur le port du hidjab par des élèves, les " dérapages et présentations stéréotypées de la communauté musulmane ont abondé dans le discours provenant de la société civile (lettres et lignes ouvertes, positions de groupes d'enseignants, de féministes ou de nationalistes "de la base"㡠 » (Mc Andrew, 2002医: 134). L'islam et, souvent, la communauté musulmane québécoise ont été présentés comme une menace pour la démocratie et l'égalité des femmes et l'islam assimilé au fondamentalisme et au terrorisme implicitement, sinon ouvertement (Mc Andrew, 2002医 : 139). Un autre conflit illustre cette attitude d'intolérance de certains Québécois. En 1997, la Loi 118 proposa des cours de «culture des religions du monde䂑 " dès le niveau primaire. Le Mouvement catholique et des groupes évangélistes du Québec se mobilisèrent contre cette proposition au nom de la nécessité d'enseigner aux élèves « leur religion 
présenter d'autres univers religieux. Ils obtinrent gain de cause医: les cours en question seront offerts uniquement au niveau IV du cycle secondaire.

Des sondages sur la perception des minorités culturelles sembleraient pourtant attester actuellement d'attitudes plus ouvertes au Québec qu'ailleurs au Canada. Au début 2002, selon un sondage de la société Environics, 68 㞟\% des Québécois répondaient par l'affirmative à la phrase䂑: "Je suis confortable dans les situations impliquant des personnes de différentes races医 》 et ce pourcentage était le plus haut recueilli au Canada. Seulement $14{ }_{20}^{[\mathrm{D}} \%$ pensaient que les minorités ethniques avaient trop

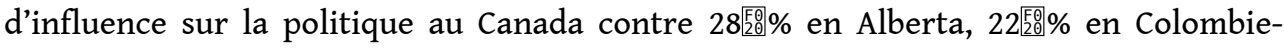
Britannique et 19 医\% en Ontario, et 49 医\% des Québécois estimaient que les mêmes minorités n'avaient pas assez d'importance dans ce domaine contre 39 棉\% en Ontario et 35医\% en Colombie-Britannique et en Alberta (The Gazette, 2002医: D3). De plus, 63医\% des Québécois n'acceptaient pas l'idée que les personnes d'origine européenne étaient discriminées au Canada. Mais quand la mention de minorités particulières est introduite, cette image d'ouverture se modifie.

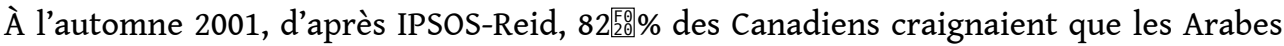
et les Musulmans deviennent la cible de préjugés mais, selon un autre sondage d'IPSOSReid en août 2002, 45覧\% des Québécois, 37国\% des Albertains, 33覧\% des Ontariens et 22茞\% des habitants de la Colombie-Britannique étaient d'accord avec l'énoncé attentats du 11 septembre 2001 m'ont rendu plus méfiant à l'égard des personnes d'origine arabe ou des musulmans venus du Moyen-Orient監 » et, de fait, en septembre 2002, d'après un sondage Léger Marketing, 33 avoir entendu des commentaires racistes à l'égard des musulmans et Arabes. Cet amalgame entre islam et violence semblait plus fort au Québec. En juillet 2002, selon un sondage CROP sur la croyance religieuse auprès de personnes de 16 à 35 ans, 76[臨\% des répondants québécois et 55 覧\% des autres répondants canadiens affirmèrent que les

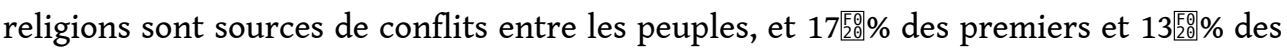
seconds que l'islam favorise des relations conflictuelles (Le Devoir, 22 juillet 2002). En novembre 2002, un sondage Maclean's magazine, Global TV et The Citizen indiqua que 44 医\% des Canadiens voulaient une réduction de l'émigration des pays islamiques, comparativement à 49 區\% un an auparavant. Mais le pourcentage le plus élevé fut

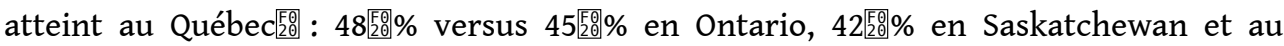
Manitoba, $43 \%$ dans les provinces maritimes, 39憕\% en Colombie-Britannique et 35覧\% en Alberta ${ }^{13}$.

Enfin, à la mi-mars 2003, soit la semaine du début de la guerre anglo-américaine contre l'Irak, 70医\% de 2002 Canadiens interrogés estimaient que l'intolérance à l'égard des

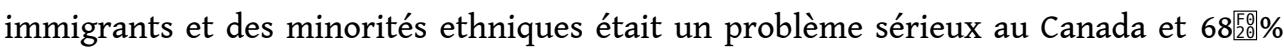

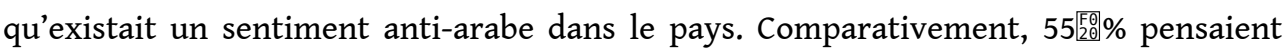
que les personnes dites noires subissaient une discrimination et 54 [F。 $\%$ que l'antisémitisme était présent. Par ailleurs, 30[0]\% dirent que les personnes d'origine arabe projetaient une image négative, comparativement à 38 医 $\%$ dans le cas des

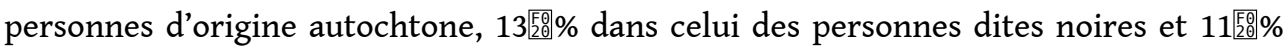
dans celui des personnes d'origine juive (Jayoush, 2003). Dans ce cas, le pourcentage des Québécois avançant que les personnes d'origine arabe projetaient une image négative

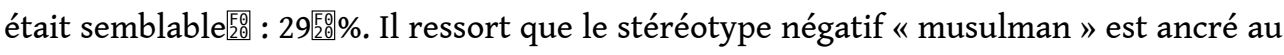
sein d'une fraction de l'opinion publique canadienne, notamment québécoise. Des organisations, notamment certains médias, l'alimentent. 


\section{La couverture médiatique} pays islamiques ou des populations, groupes ou individus de confession musulmane a donné lieu à une abondante couverture médiatique destinée au grand public occidental, notamment à propos du conflit israélo-palestinien, des guerres au Liban, en Bosnie, au Kosovo, du Golfe (1991, 2003), au Caucase, en Afghanistan, en Irak et des attentats de septembre 2001. Cette couverture, comme d'autres de l'actualité, tend à omettre toute analyse sociopolitique, historique et présente, des conflits décrits (Helly, 2002). Elle reproduit fréquemment l'archétype orientaliste (Saïd, 1978) d'une distance insurmontable entre l'islam et les autres religions monothéistes et entre l'évolution des cultures et pays occidentaux et musulmans. Karim (2000) a décrit comment le traitement par des médias canadiens d'actions terroristes menées par des Musulmans durant les années 1980-1990 reproduisait cet archétype.

Cette couverture médiatique de faits concernant des pays ou des résidents musulmans depuis septembre 2001 est encore peu documentée mais elle constitue un des sujets de critique les plus importants des organisations musulmanes. Le principal reproche adressé est la référence constante à l'islam pour qualifier des positions et actions

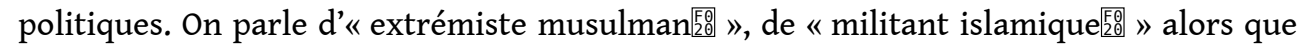
toute qualification religieuse est omise quand on traite de similaires positions ou actions de personnes d'autres religions. Lors d'une entrevue (Weld, 2003), Wahida Valiante, vice-présidente du Congrès islamique canadien (CIC), explique㬐: « We never refer to those involved in the Northern Ireland conflict as Catholic terrorists. In dealing with Bosnia and Kosovo there were Christian and Orthodox terrorists but they were never called that, though there was much discussion of Muslim Bosnian terrorists".

Selon une enquête de CAIR-CAN auprès de 296 résidents canadiens de confession musulmane en 2001, 56 devenue plus biaisée à l'égard de l'islam et des Musulmans après septembre 2001 et

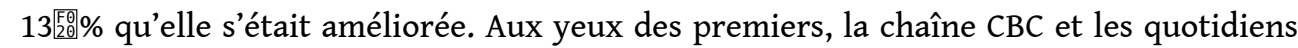
Toronto Star et Globe and Mail étaient les médias les plus objectifs alors que les journaux National Post et Ottawa Citizen et la chaîne Global s'avéraient les plus hostiles à l'islam (Media communique, 9 mai 2002医 ; Alternative Perspective, septembre 2002). Et les 181 participantes à des groupes de discussion sur la condition des «Canadiennesmusulmanes監 ” (Hussain, 2002區: 28) s'entendirent pour dire que CBC, chaîne de télévision publique nationale en anglais, avait présenté une information exacte et équilibrée les jours ayant suivi les attentats du 11 septembre 2001, notamment à travers ses programmes The National et The Passionate Eye.

Le CIC publie depuis 1998 une étude annuelle, Anti-Islam in the Media, qui évalue la couverture de faits liés à l'islam par huit journaux canadiens (Globe and Mail, La Presse, National Post, Ottawa Citizen, The Gazette, Toronto Star, Toronto Sun, Winnipeg Free Press). Chaque étude recense les articles contenant des présentations ou termes biaisés ou offensants à l'égard de musulmans et attribue un poids à ces termes selon deux

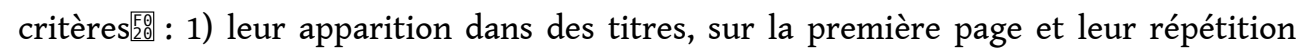
dans le même article原; 2) le taux de circulation du journal (Congrès islamique canadien, 2002棉 : 10). Le 23 mars 2003, le Congrès islamique canadien s'est vu attribuer 
un prix d'excellence par un organisme public, la Fondation canadienne des relations raciales, pour sa dernière étude, Anti-Islam in the Media 2002.

Alors qu'en 1999 le National Post était classé le journal le moins biaisé et le Globe and Mail le journal le plus biaisé14, depuis 2000 le National Post demeure le quotidien le plus hostile à l'islam et aux musulmans alors que le Globe and Mail semble au fil des années avoir éliminé les termes biaisés pour se retrouver en 2002 parmi les publications les moins condamnées par le CIC, avec le Winnipeg Free Press. Toutefois, on constata un regain de stéréotypes anti-musulmans du 12 septembre au 28 novembre 2001圈: les

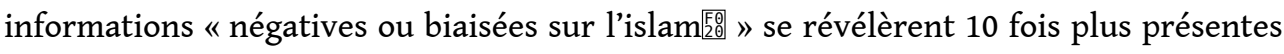
que les mois précédents dans le Toronto Star, 18 fois plus dans le Globe and Mail et 22 fois plus dans le National Post (Congrès islamique canadien, 2001). Une même conclusion a été tirée de l'étude du Vancouver Sun (Enns, 2002).

La position très négative du National Post tient à son appartenance à une chaîne de médias (CanWest Global) dont les propriétaires jouent d'autoritarisme éditorial et affichent leurs positions anti-musulmanes et en faveur du gouvernement Sharon en Israël. Leurs journaux, Ottawa Citizen, The Gazette et National Post, détiennent depuis 2000 les trois premiers rangs des journaux les plus défavorables aux musulmans et traitent plus de la montée de l'antisémitisme que de la discrimination subie par les musulmans (Congrès islamique canadien, 2002䜿: 13). The Gazette a néanmoins traité ces deux sujets en une série d'articles en septembre 2002.

Comme exemples de biais ou d'incohérence malveillante par le National Post, un article du 15 août 2002, Attacking Infidels: Terrorists Target the West for only one reason: its religious values, cite à l'appui de sa thèse l'assassinat de 400 personnes en Algérie durant la période du jeûne en 2001, et des phrases écrites par George Jonas, la première en septembre 2001, la seconde en mars 2002澄 : " From the beginning, Western attempts to

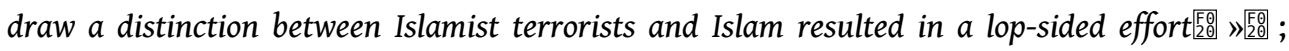
"The terrorist enemy has no armies to send against us; it has to penetrate our perimeter through fifth columnists㞔 》 (Elmasry, 2002).

En 2002, le CIC et Islamic Society of North America (ISNA) ont réalisé une recherche pilote sur les journaux télévisés du soir de trois chaînes de télévision, CTV, Global et $\mathrm{CBC}$, durant 60 jours. $\mathrm{CBC}$ fut classée comme la chaîne présentant le plus de références négatives à l'islam. La couverture par des stations de radio n'a pas encore été étudiée, mais certaines ne semblent pas étrangères à l'archétype anti-islam. Le 8 juillet 2002, lors d'une émission de radio (CFRA 580 AM, Ottawa), un journaliste, M. Green, déclara医: : North America - democracy - the Christian faith is under attack - not just in North America, but elsewhere around the world櫃 " et la menace "most exclusively comes from Muslim men[0 ॠ. Plainte a été portée au Conseil de la radiodiffusion et des télécommunications canadiennes par CAIR-CAN.

Au Québec, des auteurs (Piché et Djerrahian, 2002) ont voulu savoir si les attentats de septembre 2001 avaient porté des journalistes franco-québécois à favoriser un amalgame entre terrorisme et immigration. Ils ont analysé 78 articles parus dans $L a$ Presse et Le Devoir entre le 11 septembre et le 31 octobre 2001 et concluent que, si « le consensus sur l'immigration s'était rompu après le 11 septembre ${ }^{15\left[{ }_{20}\right.}$ ", « la plupart des publications demeurent du côté de la modération臨 », et ils n'estiment pas que la lutte contre le terrorisme conduise à un réexamen de la loi d'immigration (ibid.娣 : 82), car un contre argument était souvent présenté par les journalistes à ce propos, la pression des États-Unis à voir un tel réexamen. Selon une autre analyse des articles de La Presse et Le 
Devoir (et de Libération comme «journal écho[ (Pietrantonio, 2002), les attentats furent décrits comme une conséquence quasi inévitable de rapports de pouvoir inégaux. Par ailleurs, les chaînes francophones de télévision Radio-Canada et RDI ont couvert de manière équilibrée les événements dans les pays islamiques depuis septembre 2001 et présenté des émissions d'information sur ces pays.

\section{L'école} matériel scolaire canadien. Au Québec, à partir d'une analyse de contenu des manuels rédigés durant la deuxième moitié des années 1970, Mc Andrew avait pointé en 1985 « le dernier racisme légitime $\left[\mathbb{E}_{0}^{\mathrm{E}}\right.$ ». Alors que les manuels parlaient de solidarité avec les luttes tiers-mondistes et des Noirs américains et que Malcolm X, Allende, Mao Ze Dong ou Castro étaient présentés très positivement, les luttes arabes pour l'émancipation

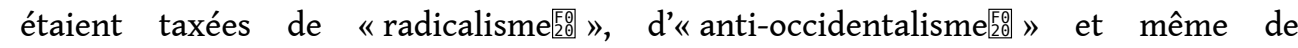
«fanatisme 20 " affrontements avec Kadhafi donnaient le ton (Mc Andrew, 2002医 : 134). De plus, la population arabe de confession maronite établie de longue date au Québec n'était pas mentionnée et le stéréotype du TV Arab dominait (Shaheen, 1984, 2001), soit les images de riche cheikh, de terroriste dangereux, de paysan inculte ou de noble Touareg. Ce profil négatif avait apparemment son effet auprès de la clientèle étudiante selon une enquête de l'Association québécoise des organismes de coopération internationale (AQOCI) en 1981 auprès de quelque 200 étudiants francophones de collèges d'enseignement général et professionnel (cégep) à qui l'on demanda d'attribuer diverses qualités et défauts à dix groupes ethniques ou nationaux. Ils évaluèrent plus positivement les anglophones et des groupes ethniques anglicisés ou anglophiles (juif, allemand) et placèrent clairement en bas de liste les groupes noir et arabe, ce dernier étant dit « malhonnête, sournois, cruel et râleur les années 1980 les manuels scolaires furent réformés mais la présentation des pays arabes et asiatiques, de leur histoire et de leur situation internationale depuis vingt ans demeure défaillante.

\section{Des conflits autour des lieux de culte}

Depuis 2001, des disputes autour de la fondation de lieux de culte illustrent le malaise et l'hostilité qui entourent la religion musulmane au Canada. Dans les régions montréalaise et torontoise où résident une large part de la population musulmane canadienne, pareille fondation n'avait posé de difficultés durant les années 1970-1980 alors que de nombreux lieux de culte étaient ouverts par des immigrés arrivant du Pakistan et du Moyen-Orient. Mais, à partir des années 1990, des conflits apparaissent. Dans la métropole montréalaise, une demande d'ouverture d'un lieu de culte musulman fait l'objet d'un conflit depuis 1995, une autre demande a été rendue inopérante et des dossiers d'agrandissement de lieux de culte existants ne trouvent pas d'aboutissement. Il en est de même à Toronto où, en 1995, un conflit très publicisé a entouré l'ouverture 
d'un lieu de culte dans East York et où la construction d'un dôme sur une mosquée pakistanaise en 1998-1999 a provoqué débats et disputes (Isin and Siemiatycki, 2002). Comme illustrations, nous présenterons des cas en cause actuellement dans la région montréalaise, dont l'un ne concerne pas une zone résidentielle où la création d'un lieu de culte, d'une synagogue ou d'une mosquée par exemple, est souvent refusée au nom de son achalandage.

Depuis 1995, un conflit larvé est le fait d'une municipalité particulièrement hostile à l'ouverture d'une mosquée à Dollard-des-Ormeaux, une zone résidentielle aisée et anglophone de l'Ouest de lîle de Montréal. La Fondation internationale Az'zahra, une organisation chiite multiethnique, voulut y construire un centre culturel incluant une salle de prière. Elle reçut l'avis favorable du Service d'urbanisme, mais le Conseil municipal changea le zonage en 1997 afin d'éviter la construction. Le projet fut abandonné en 1998 et l'organisation acheta dans la même municipalité le site d'une ancienne synagogue, lieu de culte reconnu selon le règlement de zonage, et ouvrit un centre culturel et religieux en 1999. Elle ne reçut pas de permis d'exercice de la municipalité pour les nouvelles activités de ce centre alors que le Conseil municipal en avait été avisé (Daher, 2002). En 2001, en raison de difficultés financières causées entre autres par la crainte des fidèles de faire des dons à des organisations islamiques à la suite de la Loi anti-terroriste, elle vendit le bâtiment à une organisation sunnite, le Centre islamique canadien, qui, elle aussi, n'a toujours pas reçu d'autorisation légale d'exploitation.

Le conflit fut plus abrupt et ouvert dans le cas de la création d'un centre culturel et religieux ismaélien (Jamaat Khana). Cet établissement pouvant accueillir 800 à $1{ }_{200}^{[6000}$ fidèles aurait été construit sur un terrain du centre de la municipalité de Brossard. Le projet fut bloqué en août 2002 sous la pression de 506 résidents. Ils signèrent une pétition s'opposant à une proposition de la municipalité de modifier le zonage afin de

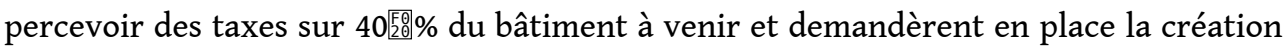
d'un parc public. Le zonage en vigueur depuis 40 ans n'avait jamais été modifié et permettait 100 [Е arguments avancés (La Presse, Forum, lundi 12 août 2002)医 : «Je n'aime pas vivre avec du monde différent. Cela ne fait pas de moi un monstre, ni un taréÉ⿴囗玉 : seulement

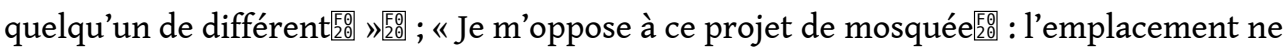
s'y prête pas. Sa valeur commerciale est à mon avis trop importante pour qu'on l'utilise à des fins religieuses, que la religion soit musulmane, catholique, bouddhiste, etc. "Selon les opposants, le terrain doit avoir une vocation neutre et ne pas servir à des

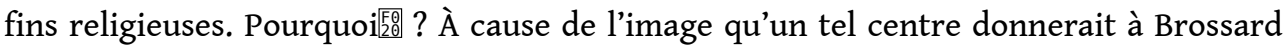
qui est pourtant une ville largement multiethnique医? L'argument ne tient pas la route. 送 " Vu le malentendu entre les résidents et le Conseil municipal sur les subtilités du changement de zonage et un débat public acerbe, le Conseil émit un moratoire de trois mois sur le dossier. L'option d'achat du terrain par l'association musulmane demanderesse venant à échéance avant la fin du moratoire et le vendeur refusant de la prolonger, l'association abandonna le projet. Aucune suite n'a été donnée au projet de construction d'un parc public. Rappelons que la même association ismaélienne a sans opposition établi depuis vingt ans des centres religieux dans d'autres municipalités du Québec医: Ville Mont-Royal, Laval, Sherbrooke, Granby et Québec. Enfin, un projet d'agrandissement d'un lieu de culte à Pierrefonds, Makka al Mukarramah, déposé en 1999 et approuvé par le Conseil consultatif d'urbanisme, n'a toujours pas reçu l'aval du 
Conseil municipal qui argue de problèmes de circulation occasionnés par la venue de quelque 250 fidèles. Seul un projet d'agrandissement est à l'œuvre, celui de la mosquée al-Islam à Ville Saint-Laurent, conçu en 1988 et autorisé il y a plusieurs années.

\section{Les fondements des stéréotypes négatifs}

61 La discrimination subie par les musulmans au Canada se distingue de celle, connue des populations musulmanes des autres sociétés occidentales par sa moindre incidence de crimes haineux consistant en des violences physiques contre des personnes. Mais difficultés d'accès au marché du travail, ciblage ethnique par des mesures antiterroristes, polémiques sur l'ouverture de lieux de culte et le port du foulard à l'école,

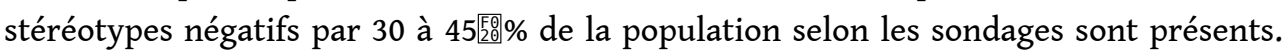
Et, comme dans d'autres pays, hormis les États-Unis, les crimes haineux ont diminué depuis 2002. Par contre, des dynamiques qui expliquent la prégnance de stéréotypes anti-musulmans au Canada actuellement, trois sont spécifiques à ce pays.

La première dynamique est similaire dans d'autres pays. Elle ressort des images négatives divulguées en Occident à propos de l'islam et observables au Canada dans des médias, des conflits locaux sur des accommodements culturels et des sondages d'opinion publique, soit l'assimilation entre islam et terrorisme, la vision de l'islam comme religion intolérante, voire violente, et source de conflits et les idées d'une impossible implantation de la démocratie dans les pays musulmans et d'une oppression des femmes dans la loi islamique.

Une seconde dynamique relève de l'histoire de la population de confession musulmane, fort différente de celle des populations musulmanes européennes et des autres minorités importantes immigrées du Canada. L'établissement récent de la majorité de ses membres, soit depuis les années 1990, explique son absence sur la scène politique et au sein du personnel des médias et sa très faible capacité de pression politique, faits qui facilitent le développement d'un stéréotype des musulmans canadiens comme fermés sur eux-mêmes, indifférents à la société canadienne et tournés vers la vie de leurs pays d'origine. Une forte fragmentation ethnique, nationale, religieuse et l'absence de soutien de programmes du gouvernement fédéral aux associations issues de la population de confession musulmane durant les années 1990 expliquent aussi sa faible structuration communautaire et sa faible mobilisation politique. Ces déficiences l'empêchent, comme communauté, de mettre à profit des dispositions canadiennes favorables aux minorités culturelles et religieuses, telles que l'obligation juridique d'accommodement raisonnable, l'invocation permanente par les autorités politiques de l'unicité et des bienfaits de la Politique du Multiculturalisme et le recours aux tribunaux pour cause de discrimination.

Une autre dynamique particulière caractérise la société québécoise où se concentre plus d'un quart des musulmans canadiens. Là les débats et conflits publics sur l'islam sont plus présents en raison de plusieurs faits. Un mouvement «laïque医 》 militant adhère à l'interprétation française, anti-religieuse, de la laïcité et considère la religiosité comme un trait culturel archaïque. Un courant ethnonationaliste voit dans l'islam et toute différence minoritaire marquée une menace à une identité nationale québécoise ancrée dans une religion chrétienne tandis que les mouvements féministes hostiles à l'islam sont influents. Enfin, l'opinion publique provinciale reconnaît généralement le droit des Palestiniens à un État et trois populations concernées par le 
débat sur l'islam sont fort présentes, celle d'origine arabe, dont maghrébine, et celles d'origine pakistanaise ou juive ${ }^{16}$. La mobilisation et les tensions entre les activistes de ces groupes, notamment en milieu étudiant, sont élevées et ont donné lieu à des incidents violents dans les locaux de l'Université Concordia ${ }^{17}$ à propos du conflit israélo-palestinien. Aussi le débat sur la situation au Moyen-Orient et sur l'« islam医 » en général est-il plus présent sur la scène publique québécoise qu'ailleurs au Canada. $\mathrm{Au}$ Canada anglais, les débats sur l'immigration concernent plutôt les niveaux d'entrées annuelles, les droits des immigrants et les flux en provenance de l'Asie, de la Chine particulièrement (Li, 2003) et les polémiques sur l'islam opposent des journalistes, des propriétaires de médias et des membres de la communauté juive. Ces derniers ont, par exemple, reproché à $\mathrm{CBC}$, chaîne de télévision publique, de désigner des membres du Hamas comme des militants extrémistes et non comme des terroristes ou encore d'ignorer la recrudescence de l'antisémitisme au Canada et ailleurs en Occident

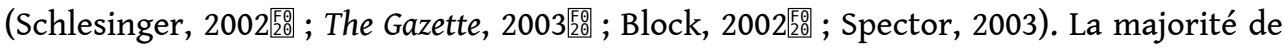
la communauté juive considère la lutte palestinienne comme une menace mortelle pour Israël et assimile islam et terrorisme, et pour le Congrès juif canadien, identité juive et État d'Israël sont synonymes.

Enfin, une quatrième dynamique, déterminante à nos yeux, tient à la proximité géographique des États-Unis, premier allié politique, militaire et économique ${ }^{18}$. D'une part, nombre de médias canadiens, notamment anglophones, s'alimentent pour leur couverture des événements au Moyen-Orient et de la lutte anti-terroriste auprès de médias américains (Maybee, 1980憕 ; Karim, 2000憕 : 14). D'autre part, depuis septembre 2001, les États-Unis exercent des pressions pour que les politiques canadiennes de contrôle des frontières, d'immigration, d'asile politique et de sécurité, ainsi que les positions internationales du gouvernement canadien, s'alignent sur les leurs. Ils insistent particulièrement sur la surveillance de la population musulmane et accusent le Canada de laxisme en la matière. Aussi le gouvernement fédéral a-t-il tendance à être silencieux sur tout dossier relatif à cette population et peu attentif à la discrimination à son égard ${ }^{19}$.

Sur ce point, le Premier ministre du Canada s'est rendu dans une mosquée d'Ottawa après les attentats en septembre 2001 pour « réaffirmer que l'islam n'a rien à voir avec

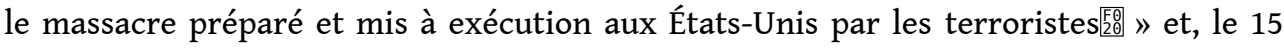
novembre 2001, il a déclaré au Parlement qu'il fallait consacrer des efforts pour lutter contre la discrimination tout autant, sinon plus, que contre le terrorisme. Depuis lors, à l'égal de tout autre membre du gouvernement et du Programme du multiculturalisme, il est demeuré silencieux et inactif, sinon pour soulever une controverse à l'automne 2002 lorsqu'il déclara que les fréquentes humiliations des pays arabes par les pays occidentaux pouvaient avoir conduit aux attentats aux États-Unis en septembre 2001.

Aussi, face à cette inaction, les 296 répondants à un sondage de CAIR-CAN durant l'été 2002 critiquèrent le Premier ministre pour «son manque d'actions claires contre la vague de haine envers les musulmans[熙 》 et près des trois quarts qualifièrent de défaillants ses liens avec la communauté musulmane (communiqué CAIR-CAN, 5 septembre 2002). Des exemples de cette indifférence des autorités publiques furent donnés par le président de la Fédération canado-arabe lors du colloque Policing in a Multicultural Society à Ottawa en 2003医 : «Why did the Justice department, after agreeing to work with us on 11 specific concerns, then walk away? Why did the solicitor-general, after expressing empathy with our plight, then refuse to help us monitor abuses of the Anti-Terrorism 
Act by law enforcement agencies? Why did the minister of foreign affairs leave the travel advisory to the U.S. when Arab and Muslim Canadians travelling there continue to face humiliation and are subjected to treatment normally reserved for charged criminals? Why did the Ontario public safety minister condone the racial profiling taking place at the U.S. border against his own citizens䂑? Why is it that the mayor of Canada's largest city, whose logo (sic) is "Diversity is Our Strength" never spoke out against the victimization of Arabs and Muslims postSept. 11 ? 覧》(Khouri, 2003).

Et R. Khouri de conclure㞔: « By and large, Arab and Muslim Canadians were left on their own, having to explain themselves and prove their loyalty ; defend their religion and demonstrate its goodness; and at times hide their ethnicity and deny their heritage in a bid to escape scrutiny頤 》.

\section{Conclusion}

Face à ce constat, une forte désillusion quant à son statut dans la société canadienne s'est installée au sein de la population musulmane depuis septembre 2001. En effet, ce

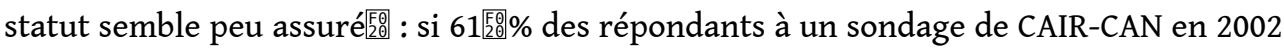
dirent avoir fait l'expérience d'actes de gentillesse ou du support d'amis ou de collègues d'autres confessions depuis septembre 2001, une proportion égale (60\%), soit souvent les mêmes personnes, mentionna l'expérience d'attitudes négatives ou ouvertement discriminatoires. Un tiers des répondants déclara aussi que leurs conditions de vie avaient empiré, qu'ils se sentaient non acceptés des autres Canadiens et étaient inquiets pour leur sécurité et celle de leur famille (communiqué CAIR-CAN, 5 septembre 2002). Lors d'un autre sondage au printemps 2002,41 医\% des 253 répondants, d'origine arabe et de diverses confessions, estimaient que les Canadiens "n'aiment pas les

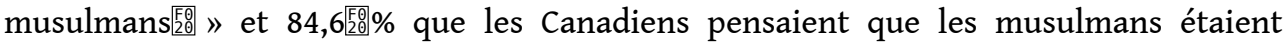
intrinsèquement violents (Fédération canado-arabe, 2002圈: 11).

Ces données questionnent les politiques canadiennes de respect et de promotion du pluralisme culturel, comme celles des autres provinces canadiennes et des métropoles torontoise et montréalaise. $\mathrm{Si}$, lorsqu'un groupe ethnoculturel devient la cible attestée de pratiques et d'attitudes hostiles relativement répandues, ces politiques se révèlent sans moyens de réduire ces manifestations discriminatoires, quelle est la réalité de leur message et de leur objectif d'équité et d'égalité de tous監?

Certes, à la différence d'autres pays occidentaux, aucun parti politique canadien n'a appelé à un ostracisme des résidents de culture islamique, ni, contrairement à une partie de l'opinion publique, à un arrêt de l'émigration provenant des pays islamiques.

BIBLIOGRAPHIE

ABDELKARIM Riad Z. (2003) Surge in Hate crimes Followed by Official U.S. Targeting of Muslim, Arab Men, The Washington Report on Middle East Affairs, avril. 
ANGUS-REID GROUP (1991) Attitudes About Multiculturalism and Citizenship, Ottawa, Multiculturalism and Citizenship Canada, juillet.

BEL HASSEN Amel (2002) De l'insertion professionnelle des immigrant5医 : le cas des Maghrébins, Vivre Ensemble 11 (38), p. 6.

BLOCK Irwin (2002) Can West chief attacks « cancer医 》 in the media. Anti-Israeli bias

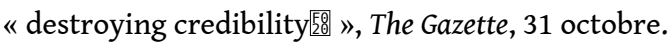

BOSSET Pierre (1989) La discrimination indirecte dans l'emploi. Aspects juridiques, Montréal, Éd. Yvon Blais.

BOSSET Pierre (1994) «Les mouvements racistes et la Charte des droits et libertés de la personne du Québec覧》, Les Cahiers de Droit 35, pp. 483-625.

BOUCHARD Hélène, Yuho CHANG et Rachid RAFFA (automne 2002-hiver 2003), Emploi et immigration dans la capitale nationale, Vivre Ensemble 11 (38), p. 8

BUREAU INTERNATIONAL DU TRAVAIL (1968) Lutte contre la discrimination dans le travail, Genève.

CICERI Coryse (1999) Le foulard islamique à l'école publique : analyse comparée du débat dans la presse française et québécoise francophone (1994-1995), Montréal, Immigration et Métropole, Université de Montréal (mémoire de maîtrise).

CONGRÈS ISLAMIQUE CANADIEN (2001) Anti-Islam in the Media 2001, in www.canadianislamiccongress.com

CONGRÈS ISLAMIQUE CANADIEN (2002) Anti-Islam in the Media 2002, in www.canadianislamiccongress.com

CONSEIL DES RELATIONS INTERCULTURELLES ET DE L'IMMIGRATION (1993) La gestion des conflits de normes par les organisations dans le contexte pluraliste de la société, Montréal, Le Conseil, 109 p.

CREPEAU François (1996) « Le migrant dans l'ordre juridique canadien[联 》, Hommes et Migrations 1220, pp. 15-24.

DAHER Ali (2002) Les lieux de culte islamique en immigration : le cas montréalais, rapport de recherche, Montréal.

DIENE Doudou (2003) Special Report. Situation of Muslim and Arab peoples in various parts of the world in the aftermath of the events of 11 September 2001 (ECN4/2003/23), New York, Nations Unies, 3 janvier.

DUCHESNE André (2001) La Presse, 20 septembre.

ELMASRY Mohamed (2002) « Words of Intolerance医 》, The Gazette, 5 septembre.

ENNS Aiden S. (2002) Assessing the Coverage of Islam in the Vancouver Sun After September 11, Vancouver, University of British Columbia, M.A.

FÉDÉRATION CANADO-ARABE/CANADIAN ARAB FEDERATION (2002) Arabs in Canada. Proudly Canadian and Marginalized, Ottawa, avril.

Groupe de travail sur la participation des minorités visibles dans la fonction publique fédérale (2000) Faire place au changement dans la fonction publique fédérale, Ottawa.

HELLY Denise (2002) Occidentalisme et islam Renaud, Guy Bourgeault et Linda Pietrantonio Les événements du 11 septembre et les orientations de 
recherche en relations ethniques, Montréal, Presses de l'université de Montréal, septembre, pp. 229-252.

HURST Lynda (2002) Who is High Risk医 ?, Toronto Star, 20 octobre.

HUSSAIN Samira (2002) La Voix des Canadiennes-Musulmanes, Mississauga, Ontario, Le Conseil Canadien des Femmes Musulmanes, $41 \mathrm{p}$.

ISIN E.F. and M. SIEMIATYCKI (2002) Making Space For Mosques医: Struggles For Urban Citizenship In Immigrant Toronto, in Sherene H. Razack (ed.) Race, Space and the Law Making of a White Settler Society. Toronto, Between the Lines, pp. 185-209.

JAYOUSH Kinda (2003) « We're intolerant, Canadians say苞 », The Gazette, 22 avril, A10.

JEZEQUEL Myriam (2002) Au nom de la loi C36, Journal du barreau, 20 (34), $1^{\text {er }}$ décembre.

JOLY Jacques et Michel DORVAL (1993) Sondage sur l'opinion publique québécoise à l'égard des relations sociales et interculturelles, Montréal, ministère des Communautés culturelles et de l'Immigration, $76 \mathrm{p}$.

KARIM Karim H. (2000) The Islamic Peril医: Media and Global Violence, Montreal, Black Rose, 204 p.

KHOURI Raja (2003) Can multiculturalism survive security agenda軞 ? Toronto Star, 9 mars.

KUNZ J.-L., A. MILAN et S. SCHETAGNE (2001) Inégalité d'accès : profil des différences entre les groupes ethnoculturels canadiens dans le domaine de l'emploi, du revenu et de l'éducation, Ottawa, Conseil canadien de développement social (étude préparée pour la Fondation canadienne des relations raciales).

LEDOYEN Alberte (1992) Montréal au pluriel. Huit communautés ethnoculturelles de la région montréalaise, Québec, Institut québécois de recherche sur la culture, 318 p.

LENK Helle-Mai (2000) The Case of Émilie Ouimet㹂: News Discourse on Hijab and the Construction of Québécois National identity, in Agnes Calliste and George J. Sefa Dei (eds) AntiRacist Feminism, Halifax, Fernwood Publishing, pp. 73-90.

LI Peter (2003) « The Place of Immigrants医: Politics of Difference in Territorial and Social Space㞔 》, Conférence, Congrès des Études Ethniques Canadiennes, Banff, septembre, manuscrit, $19 \mathrm{p}$.

LUBUTO MUTOO Valentin (2001) Discrimination raciale en milieu de travail dans la région métropolitaine de Québec, Montréal, La Ligue des droits et des libertés du Québec.

MAC DONALD Gayle (2003) Jewish group plans rally against CBC, National Post, January 14, page A2.

MAKIN Kirk (2003) Police engage in profiling, chief counsel tells court, Globe and Mail, 18 janvier.

MAYBEE Jack (1980) Reporting the Third World医 : The Canadian Angle, Carleton Journalism Review, Winter医: $: 6-9$.

MAC ANDREW Marie (1985) « Le traitement du monde arabe dans les manuels scolaires

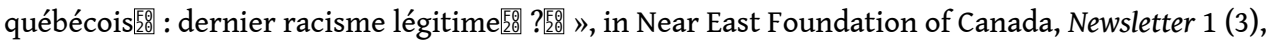
novembre.

MAC ANDREW Marie (2002) « Le remplacement du marqueur linguistique par le marqueur religieux en milieu scolaire医 ", in Jean Renaud, Linda Pietrantonio et Guy Bourgeault (dir.) Ce qui a changé depuis le 11 septembre 2001. Les relations ethniques en question, Montréal, Presses de l'université de Montréal, septembre, pp. 131-148. 
MAC ANDREW Marie et Maryse POTVIN (1996) Le racisme au Québec : éléments d'un diagnostic, Montréal, ministère des Affaires internationales, de l'Immigration et des Communautés culturelles, $183 \mathrm{p}$.

MINISTÈRE DE L'ÉDUCATION DU QUÉBEC (1995) Module de formation à l'intention des gestionnaires, cahiers 8 et 8.1, Québec, le Ministère, 33 pages et 14 pages.

MONTGOMERY Sue (2003) « Music's over for Osama[를 》, The Gazette, 8 janvier.

NIMER Mohammed (2002) Report, The Council on American-Islamic Relations, www.caircan.ca, mars.

PENDAKUR Ravi (2000) Immigrants and the Labor Force. Policy, Regulation and Impact, MontréalKingston, McGill-Queen's University Press, 248 p.

PICHE Victor et Gabriella DJERRAHIAN (2002) « Immigration et terrorisme医: : une analyse de la presse francophone 200 ", in Jean Renaud, Linda Pietrantonio et Guy Bourgeault (dir.). Ce qui a changé depuis le 11 septembre 2001. Les relations ethniques en question, Montréal, Presses de l'université de Montréal, septembre, pp. 81-94.

PIETRANTONIO Linda (2002) « Rapports de pouvoir dans le savoir public㡠 ? Les mots de miseptembre 2001医 》, in Jean Renaud, Linda Pietrantonio et Guy Bourgeault (dir.) Ce qui a changé depuis le 11 septembre 2001. Les relations ethniques en question, Montréal, Presses de l'université de Montréal, septembre, pp. 113-127.

PROULX Jean-Pierre (1994) « La prise en compte de la diversité religieuse à l'école québécoise :

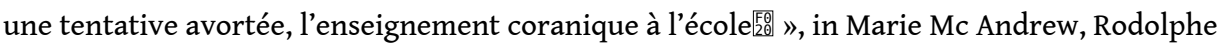
Toussaint et Olga Galatanu (dir.) Pluralisme et Éducation : politiques et pratiques au Canada, en Europe et dans les pays du Sud, Montréal, Presses de l'Université de Montréal, pp. 251-265.

SAÏD Edward W. (1978) Orientalism, New York, Vintage Books, 368 p.

(1997). Covering Islam. How the media and the experts determine how we see the rest of the world, New York, Vintage Books, 200 p.

SHAHEEN Jack G. (1984) The TV Arab, Bowling Green, Bowling Green State University Popular Press.

(2001) Reel Bad Arabs. How Hollywood Vilifies a People, New York, Olive Branch Press, 573 p.

SCHLESINGER Frank (2002) Anti-Semitism is there but it is less overt, The Gazette, 25 septembre.

SPECTOR Norman (2003) CBC news on Mideast smacks of propaganda, The Gazette, 21 janvier.

TADLAOUI Jamal-Eddine (2002) Pistes d'action pour contrer les manifestations de la discrimination raciale et de l'intolérance dans le champ de l'intégration en emploi des personnes immigrées au Canada au cours des dix dernières années, Montréal, Table de concertation des organismes au service des personnes réfugiées et immigrantes, $45 \mathrm{p}$.

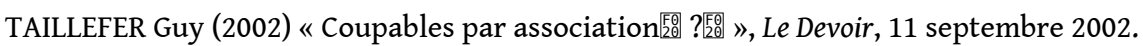

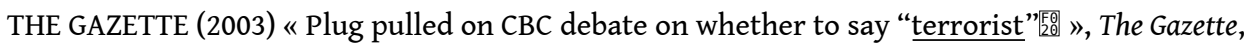
reproduisant un article de Ottawa Citizen, 16 janvier.

THE GAZETTE (2003) « Anti-Semitic incidents jump 60鍳\%咺 : B’nai Brith医 », 7 mars.

TORONTO POLICE SERVICE HATE CRIME UNIT (2001) 2001 Hate Bias Crime Statistical Report, www.torontopolice.on.ca/publications.

TORONTO POLICE SERVICE HATE CRIME UNIT (2002) 2002 Hate Bias Crime Statistical Report, www.torontopolice.on.ca/publications. 
VILLE D'OTTAWA (2002) No Hijab is Permitted Here, Ottawa (in «Permitting the hijab医 », Toronto Star - 22 décembre).

WELD Kirsten (2003) « Anti-Islamic Stereotyping in the Canadian Media. The Daily navigates the murky waters of anti-Muslim bias in Canada's newspapers[20]

ZAPPI Sylvia (2003) «2002㡠: le racisme progresse, les actes antisémites se multiplient医 》, Le Monde, 29 mars.

\section{NOTES}

1. Les données incluses dans ce texte proviennent d'une recherche subventionnée par le Conseil de la Recherche en Sciences Humaines du Canada (CRSH-IDR-2003-2005).

2. La Charte canadienne régit les relations entre particuliers et gouvernements mais non entre particuliers. Le droit civil demeure de compétence provinciale selon la Loi constitutionnelle de 1867 et les rapports privés sont régis par les chartes provinciales. La loi canadienne sur les droits de la personne (Canadian Human Rights Act) s'applique aux relations entre particuliers dans les champs de compétence fédérale (entreprises d'État, société de la Couronne) et elle traite de la discrimination.

3. Il existe trois catégories de crime haineux, définis dans la section du Code criminel sur la propagande haineuse : l'encouragement au génocide (art. 318), l'incitation publique à la haine (art. 319, par. 1) et la fomentation volontaire de la haine autrement que dans une conversation privée (art. 319, par. 2).

4. Les 64 autres crimes haineux visaient des personnes agressées en raison de leur origine culturelle, pakistanaise, somalienne, afghane, moyen-orientale, arabe, etc.

5. Ce qui exige une interprétation prudente des résultats.

6. En certains points, cette loi est similaire au Patriot Act voté en octobre 2001 aux États-Unis et au Crime and Security Act voté en Grande-Bretagne en décembre 2001.

7. Une activité terroriste est définie par la loi C-36 comme un acte commis « au nom d'un but, d'un objectif ou d'une cause de nature politique, religieuse ou idéologique » et qui « compromet gravement la santé ou la sécurité de tout ou partie de la population ».

8. Cette clause a suscité des craintes de voir des propriétés de personnes de culture islamique saisies sans preuve et les donations aux lieux de culte et autres organisations diminuer de peur de voir les fonds détournés à des fins interdites par la loi.

9. $40 \%$ des immigrés des années 1990 âgés de 25 à 54 ans détenaient un diplôme universitaire versus $23 \%$ des natifs de même âge.

10. Celles-ci sont possibles mais très coûteuses; elles requièrent des commandes spéciales de données à Statistique Canada.

11. Il s'agit d'un espace consacré par les rabbins par un procédé légal de dérogation. L'erouvrechouyot (erouv des domaines) consiste à délimiter un espace urbain (Amérique du Nord, Alsace par exemple) par un fil de fer symbolique permettant de classer l'espace urbain en espace privé : on peut y porter des objets d'un domicile à l'autre dans tout le périmètre sans déroger au chabbat (NdlR).

12. Un argument sans fondement car l'État canadien n'est pas un État laïc. De plus il accorde des privilèges aux Églises catholique et protestante.

13. En août 2002, selon un sondage de l'Association des études canadiennes (Association for Canadian Studies), $43 \%$ des répondants estimaient que le Canada acceptait trop d'immigrés des pays arabes, $40 \%$ des pays asiatiques, $24 \%$ d'Afrique, $21 \%$ d'Amérique latine et $16 \%$ d'Europe. Quand les répondants craignaient une future attaque terroriste en Amérique du Nord, 
les pourcentages augmentaient : $49 \%$ voulaient une réduction de l'immigration arabe et $47 \%$ de l'immigration asiatique.

14. Sans tenir compte du taux de circulation, La Presse était le journal le plus biaisé en 1999. En 1998, les quotidiens les plus biaisés étaient le Toronto Star, puis le Globe and Mail.

15. Un sondage à Montréal durant l'automne 2001 indiqua aussi que $83 \%$ des résidents montréalais interrogés désiraient resserrer les lois d'immigration et 77 \% accroître le contrôle aux frontières.

16. Selon un sondage de Compas Inc., $26 \%$ des Québécois et $10 \%$ des autres Canadiens estimaient que les Juifs avaient trop de pouvoir au Canada et $15 \%$ des Canadiens (dont $26 \%$ des Francophones et $39 \%$ de ceux détenant un niveau de scolarité secondaire ou inférieur) que « la persécution subie par les Juifs avant et pendant la Seconde guerre mondiale était en partie ou surtout de leur faute ", The Gazette, 25 septembre 2002.

17. Sur 30医000 étudiants inscrits dans cette Université, $10 \%$ sont de confession musulmane.

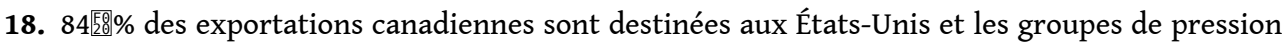
des industriels liés à ce marché ont leur importance dans les débats sur l'islam au Canada.

19. Une enquête pancanadienne sur Religion et Disrimination est envisagée depuis début 2003 par Patrimoine Canada, ministère dont relève le Programme du Multiculturalisme.

\section{RÉSUMÉS}

À la suite des attentats terroristes de septembre 2001 aux États-Unis, les actes hostiles se sont multipliés à l'égard des personnes de confession musulmane dans les sociétés occidentales. Cet article retrace les diverses formes de discrimination subies par les Musulmans au Canada autant que le permettent les données compilées avant et après les évènements de septembre 2001. Il tente aussi de repérer les fondements de cette discrimination qui s'avèreraient propres au Canada, un pays dont l'État se proclame le seul État multiculturel au monde et parmi les plus respectueux des droits des immigrés et de leurs descendants.

Current anti-islamic Trends in Canada. Following the terrorist acts in the United States in September 2001, hostility towards Muslims increases in North America and Europe. This article describes the different forms of discrimination suffered by Muslims in Canada inasmuch as the information compiled during the last two years allows it. It also attempts to describe the main factors of the hostility towards Muslims in Canada and how these factors could be peculiar to the Canadian society, which State proclaims itself the only multicultural State in the West and one of the most respectful of immigrants and cultural minority rights.

Tendencias actuales del trato que Canadá brinda al Islam. Tras los atentados terroristas de septiembre 2001 en Estados Unidos, los actos hostiles a las personas de confesión musulmana se han multiplicado en las sociedades occidentales. A partir de datos recopilados antes y después de los sucesos de septiembre 2001, este articulo retraza las diversas formas de discriminación que los musulmanes sufren en Canadá. Se trata igualmente de identificar los fundamentos de esta discriminación y cómo dichos fundamentos parecen ser específicos a la sociedad canadiense. En este país, el Estado se proclama como el único Estado multicultural del mundo y como uno de los más respetuosos de los derechos de los emigrantes y de sus descendientes. 
INDEX

Mots-clés : discrimination, islam, lieux de culte, musulmans, pratique religieuse, stéréotypes Index géographique : Canada

\section{AUTEUR}

\section{DENISE HELLY}

Professeure titulaire à l'Institut National de la recherche Scientifique, Urbanisation, Culture et Société, 3465 Durocher Montréal, Québec, Canada H2X 2C6 